\title{
DISFUNCIÓN MICCIONAL EN EL CÁNCER DE PRÓSTATA
}

\author{
Luis Prieto Chaparro.
}

Unidad de Urodinámica. Servicio de Urología. Hospital General de Elche. Elche. Alicante. España.

\begin{abstract}
Resumen.- OBJETIVO: El cáncer prostático es la neoplasia urológica que ha experimentado más cambios en la última década, habiéndose demostrado un gran aumento en su incidencia y en su diagnóstico. El manejo de los pacientes con cáncer de próstata es evidentemente multidisciplinar, participan principalmente Urólogos, pero también Anatomopatólogos especializados, Radiólogos, Bioquímicos, Oncólogos Médicos y Radioterapeutas y otros.
\end{abstract}

La larga supervivencia esperada generalmente en ellos, hace que no sea sostenible la rutinaria visita de control con PSA, no prestando la debida atención a la presencia de síntomas urinarios, amén de otros trastornos funcionales cuya repercusión afecta en gran medida a su calidad de vida.

\section{CORRESPONDENCIA}

Luis Prieto Chaparro

Unidad de Urodinámica

Servicio de Urología

Hospital General de Elche

Camino de la Almazara, s/n

03230 Elche. Alicante. (España).

prieto_lvi@gva.es
Pretendemos incidir en la disfunción miccional que acompaña a pacientes con cáncer de próstata, desde un punto de vista no oncológico y sí urodinámico y de calidad de vida. Secundariamente plantear los aspectos clínicos no trasladados a patología funcional y urodinámica ltoxicidad urinaria, toxicidad radioinducida, hiperactividad vesical...)

MÉTODOS: Revisión y puesta al día de la disfunción miccional que puede acompañar al cáncer de próstata. No solo de la incontinencia post-prostatectomía radical. Se integra la experiencia del autor en su fisiopatología, electromiografía esfinteriana y urodinámica, así como de la revisión general del tema según la bibliografía más actual.

RESULTADOS: Se ha mejorado mucho la técnica quirúrgica, proporcionando resultados de continencia muy altos, sin poderse plantear que una vía de abordaje o el empleo del robot sea superior a las demás.

Son múltiples las situaciones urodinámicas que nos presenta el cáncer de próstata. Obstrucción del tracto urinario inferior, compresiva o constrictiva, hiperactividad del detrusor, acomodación disminuida, incontinencia urinaria de esfuerzo por déficit esfinteriano, incontinencia urinaria mixta, desfuncionalización vesical. Otras situaciones no están definidas por la urodinámica.

CONCLUSIONES: Los pacientes con cáncer de próstata pueden tener actualmente una evaluación deficiente de la calidad de vida por parte del urólogo respecto a sus síntomas urinarios por prevalecer los aspectos oncológicos en su evaluación.

El cáncer de próstata muestra una gran diversidad de situaciones urodinámicas objeto de estudio, no solo la incontinencia post-prostatectomía radical.

Palabras clave: Cáncer próstata. Incontinencia urinaria. Urodinámica. Obstrucción del tracto urinario inferior. Hiperactividad del detrusor. 
Summary.- OBJECTIVES: Prostate cancer is the urologic neoplasia that has experienced more changes in the last decade, with a great increase in incidence and diagnosis. Prostate cancer patient's management is clearly multidisciplinary, with the participation of urologists mainly, but also specialized pathologists, radiologists, biochemists, medical oncologists, radiation oncologists and others.

The expected long survival makes the routine visit for PSA control non sustainable, without paying attention to the presence of urinary symptoms and other functional disorders with great repercussion on the quality of life.

OBJECTIVES: To present urinary dysfunction in patients with prostate cancer from a non oncological but urodynamic and quality of life point of view. Secondarily, to pose the clinical features not translated to functional and urodynamic pathology (urinary toxicity, radiation toxicity, bladder hyperactivity...)

METHODS: We review and update voiding dysfunction that may be present in prostate cancer patients. Not only urinary incontinence after radical prostatectomy. We integrated author's experience with its physiopathology, sphincter electromyography, and urodynamics, and a general review on the topic using the latest bibliography.

RESULTS: Surgical techniques have improved very much, resulting in very high continence rates, which do not enable to consider one approach or the use of robotics better than others.

Prostate cancer present multiple urodynamic scenarios. Constrictive or compressive lower urinary tract obstruction, detrusor muscle hyperactivity, diminished complian$c e$, stress urinary incontinence due to sphincteric deficit, mixed urinary incontinence, bladder dysfunction. Other scenarios are not defined by urodynamics.

CONCLUSIONS: Patients with prostate cancer may CUrrently receive a defective evaluation by their urologists in terms of urinary symptoms due to the prevalence of oncological features in their evaluation.

Prostate cancer shows a great diversity of urodynamic scenarios subject of study, not only post-radical prostatectomy incontinence.

Keywords: Prostate cancer. Urinary incontinence. Urodynamics. Lower urinary tract obstruction.

Detrusor muscle hyperactivity.

\section{INTRODUCCIÓN}

El cáncer prostático es la neoplasia urológica que ha experimentado más cambios en la última década, habiéndose demostrado un gran aumento en su incidencia y en su diagnóstico. La causa de este aumento de frecuencia se debe buscar en el empleo del PSA, en el envejecimiento de la población, en el aumento de la calidad de la evaluación de los enfermos y en otros factores desconocidos. Las predicciones europeas más recientes describen que el 24, $1 \%$ de todos los tumores diagnosticados en el varón son de próstata, por delante de los de pulmón. Esto supone 301500 nuevos casos diagnosticados en 2006 (1).

Actualmente el manejo de los pacientes con cáncer de próstata es evidentemente multidisciplinar, participan Urólogos, Anatomopatólogos especializados, Radiólogos, Bioquímicos, Oncólogos Médicos y Radioterapeutas. Dentro de la Urología los encargados de la patología funcional también tienen mucho que decir en la atención de estos pacientes, sobre todo por la diversidad de condiciones clínico-urodinámicas que generan. No solo se han de evaluar los aspectos oncológicos en estos enfermos. La larga supervivencia esperada generalmente en ellos, hace que no sea sostenible la rutinaria visita de control con PSA, no prestando la debida atención a la presencia de síntomas urinarios, amén de otros trastornos funcionales cuya repercusión afecta en gran medida a su calidad de vida. No solo existen en estos enfermos aspectos como la incontinencia urinaria postprostatectomía radical, muy ingeniosamente tratada con multitud de técnicas y dispositivos actualmente. Hay un espectro enorme de situaciones y condiciones clínicas y urodinámicas en estos enfermos, que va desde la simple obstrucción del tracto urinario inferior, hasta situaciones complejas de baja acomodación vesical con bloqueo funcional de la encrucijada esfinteriana.

Si se evalúa el impacto en la calidad de vida de los síntomas del tracto urinario inferior, en una población de más de 50 años, incluyendo pacientes con cáncer de próstata o hiperlasia benigna de próstata, se demuestra, que los enfermos que padecen síntomas severos, tienen un impacto muy importante en la calidad de vida. Si además consideramos el factor edad avanzada, se puede llegar a explicar una variación aproximada de la calidad de vida en aproximadamente un $30 \%$. La nicturia y la dificultad de vaciado son los datos más relevantes, de esta y otras series (2) (evaluadas mediante BPH Impact Index and Functional Assessment Cancer Therapy). Si se estudia en la población general, los factores más comúnmente asociados a la nicturia relevante, 
resultan ser para el varón el cáncer de próstata y la ingesta de antidepresivos, mientras que para la mujer son la enfermedad coronaria y la diabetes. Por otra parte, para ambos sexos, los factores asociados mas frecuentemente a la nicturia son el síndrome de piernas inquietas y la obesidad (3).

Los trastornos funcionales de la micción en estos enfermos se manifiestan a muchos niveles: desde el comienzo de la enfermedad, con la progresión, con los múltiples intervencionismos, con los tratamientos que se llevan a cabo y con las complicaciones de los mismos. No es fácil proporcionar soluciones. El urólogo debe el reconocer el deterioro de la calidad de vida de sus enfermos en lo que se refiere a los síntomas urinarios. Se ha descrito, que los pacientes tratados por cáncer de próstata localizado que han adquirido supervivencia, pueden tener generalmente una insuficiente evaluación por el urólogo de su calidad de vida (4). Esto lo demuestran en una cohorte de 1366 varones tratados por cáncer de próstata localizado mediante prostatectomía radical, radioterapia externa o braquiterapia. Evalúan la calidad de vida y los síntomas miccionales (cuestionario SF-36 y UCLA-PCI) así como la percepción del urólogo sobre la misma. Confirman que la impresión que tiene el urólogo sobre la calidad de vida y sobre los síntomas miccionales no se correlacionan con la calidad de vida que manifiestan los enfermos. Lo que prevalece es estrictamente el control oncológico de la enfermedad y las cifras de PSA, olvidando que en muchos enfermos la supervivencia está casi garantizada por un largo periodo de tiempo, quedando la servidumbre de sus síntomas miccionales, como una cosa constitucional.

En otro orden de cosas, también tiene relevancia, el origen de la información sobre la enfermedad que llega a los enfermos. Actualmente Internet proporciona mucha información sesgada, que por inadecuada o por su comprensión, puede afectar a las expectativas de los enfermos respecto a su enfermedad y a la relación con el médico. Este extremo ha sido estudiado por Steinberg (5). Se demostró, que aunque algunos videos son fuentes robustas de información, dada la trascendencia de los temas tratados, hay un desequilibrio en los videos revisados que se muestran en You Tube sobre PSA, cáncer de próstata, cirugía y radioterapia. Los autores demuestran que You Tube es una fuente inadecuada de información, para los pacientes de cáncer de próstata (5).

En esta puesta al día se aborda una panorámica actualizada de los trastornos funcionales que tienen los pacientes con cáncer de próstata por sí solo y como consecuencia de su evolución y tratamientos que se realizan en la actualidad.

\section{FISIOPATOLOGÍA}

En el varón normal la continencia urinaria depende de la integridad del mecanismo esfinteriano tanto proximal como distal. El mecanismo proximal incluye el músculo liso del cuello vesical, la uretra preprostática y los elementos de soporte de la misma. Como mecanismo distal se incluye el músculo uretral, ápex prostático, diafragma urogenital y fibras intra y extramurales estriadas de éste área (6).

Los nervios aferentes de la próstata juegan un esencial papel en la fisiopatología de la disfunción miccional tanto por el crecimiento de la glándula por el tumor como por su posible lesión con los tratamientos que se van a emplear.

El crecimiento inicial del tumor prostático no produce cambios fisiopatológicos iniciales diferentes de la hiperplasia prostática benigna hasta que adquiere un volumen suficiente o compromete el vaciado o la funcionalidad del mecanismo esfinteriano.

El crecimiento avanzado de la glándula puede comportar la obstrucción al vaciado de forma similar a la hiperplasia prostática benigna. También con la progresión caudal del crecimiento puede originar disfunción esfinteriana estableciendo tanto trastornos de vaciado como de continencia, así como establecer mecanismos que darán origen a contracciones involuntarias de la vejiga, que daría lugar a síntomas irritativos. Por otra parte, el crecimiento en volumen puede originar la disminución de la capacidad vesical funcional, ser el origen de contracciones vesicales involuntarias y también de acomodación vesical disminuida en algunos casos.

La cirugía radical de la próstata puede afectar a los nervios sensoriales aferentes procedentes de los segmentos L5-S1 y en menor parte los que llegan provenientes de D12-L2. El plexo prostático discurre por las bandeletas neurovasculares. Los nervios cavernosos llegan entre la cápsula prostática y la fascia endopélvica, emplazándose entre el ápex prostático y la base a 9-12 $\mathrm{mm}$ del diafragma urogenital. Los nervios capsulares emergen a nivel de dos segmentos, el primero a la altura de las vesículas seminales entrando en la glándula en dirección caudal y el segundo lo hace entrando póstero-lateralmente ápex.

Tras la prostatectomía radical hay que tener en cuenta fundamentalmente tres factores en relación a la continencia urinaria posterior:

- el cuello vesical y su reconstrucción,

- las bandeletas neurovasculares (su preservación o excisión) y por último 
- la existencia (y en que grado de capacidad funcional) de mecanismo esfinteriano distal.

Ante los dos primeros factores existe actual controversia. Algunos autores creen que la reconstrucción del cuello vesical participa en el mantenimiento de la continencia urinaria, situación discutida (7). En lo que se refiere a la preservación de las bandeletas neurovasculares, si influyen estas o no de forma decisiva o no en el mantenimiento de la continencia urinaria, también existe controversia: Steiner (6) afirma en su estudio que la edad del paciente, tamaño prostático, RTU previa, estadio tumoral, preservación o excisión de bandeletas neurovasculares, no tienen una repercusión significativa en el control de la continencia urinaria. Sin embargo, otros autores (7) afirman lo contrario en base a que fibras autonómicas pueden pasar por fuera del plexo periprostático e influír de forma decisiva en el mantenimiento de la continencia.

La diferente relevancia de los factores implicados en la determinación de la continencia urinaria, en pacientes sometidos a prostatectomía radical per-

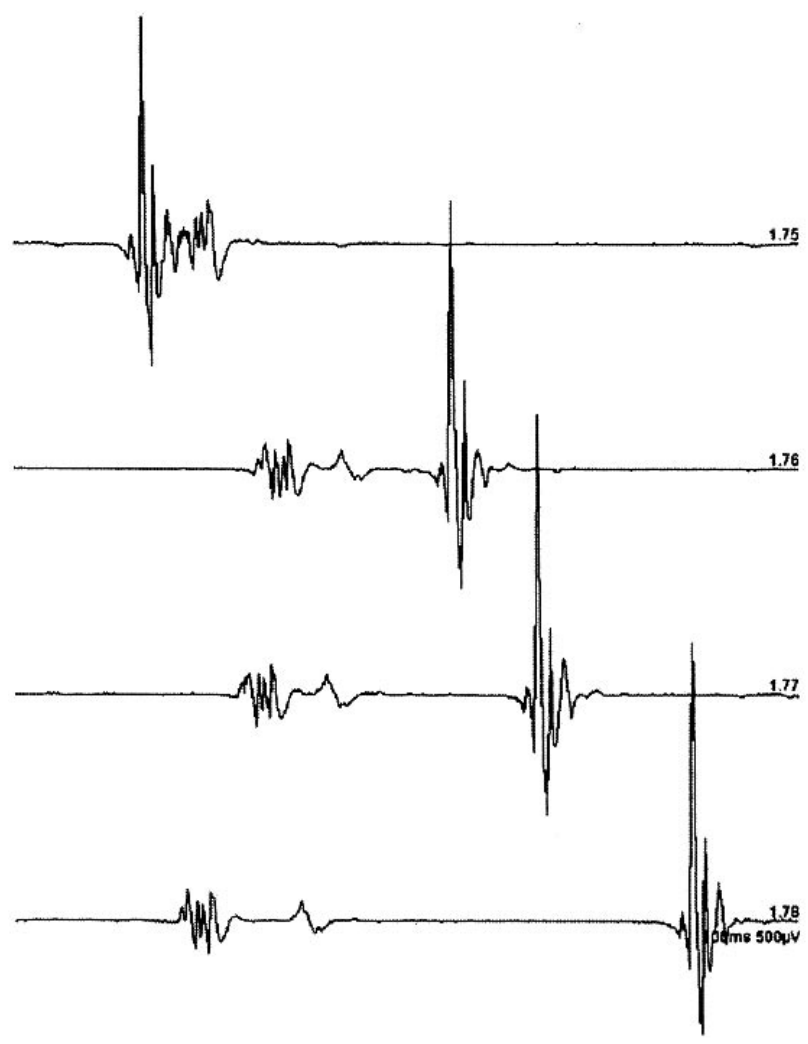

FIGURA 1. Potenciales de unidad motora reinervativos.

A). Potenciales nacientes; barido ms; amplitud: $200 \mu \mathrm{V}$;

B). Potenciales reinervativos en evolución; barrido: $100 \mathrm{~ms}$; amplitud: 200u V;

C). Potenciales reinervativos antiguos o cronificados; barrido: $100 \mathrm{~ms}$; amplitud: $500 \mu \mathrm{V}$; manecen aún en controversia. Son referidos resultados muy variables, en lo que se refiere a la valoración de la continencia urinaria en pacientes sometidos a prostatectomía radical, resultado de una no unánime evaluación metodológica, siendo de diversa índole el conjunto de factores que intervienen en los mecanismos intrínsecos de continencia urinaria. La incidencia global de la severa incontinencia urinaria posterior a prostatectomia radical se cifra en la literatura entre el 0,1 y el $12 \%(8,9,10)$. Pero estos rangos, aparte de su amplitud y dispersión, son aún más variables en función del método que valora y cuantifica funcionalmente el mecanismo esfinteriano.

La aplicación de las técnicas electromiográficas en el campo de la Urología, y en particular en la valoración de lesiones neurológicas esfinterianas ha aportado una valiosa información cualitativa (1 1), en su estudio sobre un modelo animal de experimentación le permitió afirmar que la electromiografía esfinteriana con carácter cuantitativo permite definir con mayor exactitud déficits motores y las variaciones de dichas alteraciones frente a la valoración cualitativa. Para ello han realizado electromiografía selectiva del esfínter periuretral y han comparado en diversas situaciones neurológicas la actividad electromiográfica de las unidades motoras con la existencia de fibras musculares (comprobado mediante preparaciones histológicas y morfométricas)

La electromiografía selectiva del esfínter periuretral intenta estudiar la existencia funcional documentada de mecanismo esfinteriano y establecer una posible proporcionalidad de la actividad electromiográfica de las unidades motoras, a la existencia de fibras musculares a ese nivel (12). Los reflejos electromiográficos uretrales presentes en todos los casos, con actividad disminuída frente al patrón normal reflejan una lesión incompleta de neurona motora inferior, aún cuando la existencia de potenciales polifásicos apunta la existencia de fenómenos de reinervación (Figuras 1 y 2).

El perfil uretral en el estudio de estos enfermos es controvertido por su reproductibilidad. Otros autores para evaluar la continencia basan su valoración fundamentalmente en el perfil uretral $(8,13)$, describiendo configuraciones del mismo comunes en estos pacientes, e incluso con significación pronóstica, lo que en nuestra experiencia no representó validez, ni reproductibilidad, aún realizándolo tres veces en cada paciente, quizá el perfil uretral dinámico (del que no disponemos) pudiera aportar los datos referidos.

Otros autores (8) remarcan la trascendencia de la corta longitud uretral esfinteriana, quizá como 


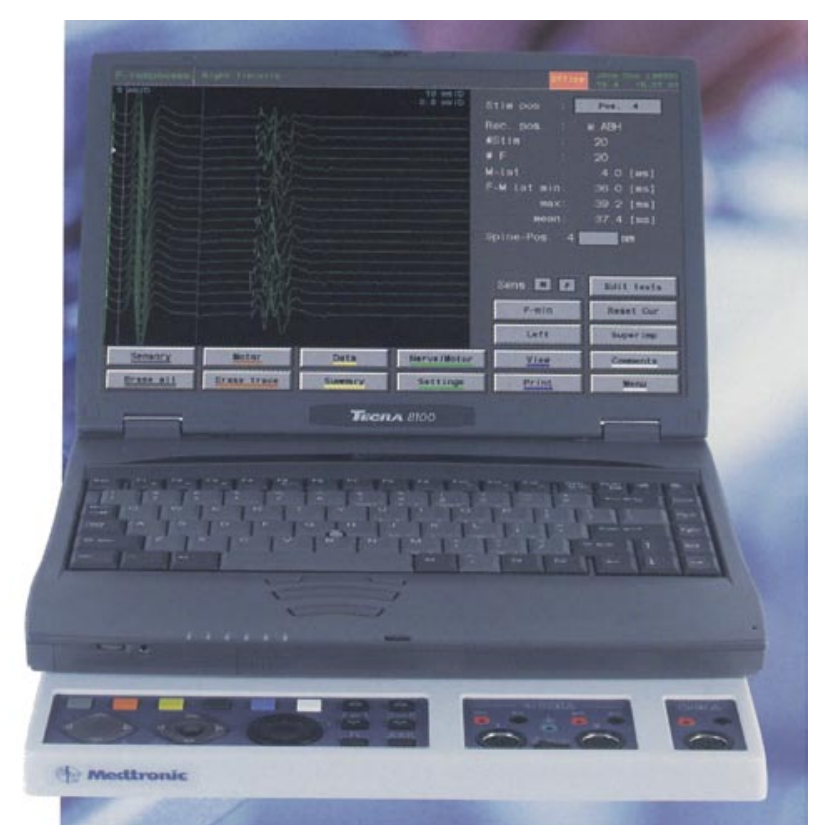

FIGURA 2. Electromiógrafo de aguja convecional. Permite obtener registros electromiográficos cuantitativos del esfínter periuretral residual obreciendo datos cuantitativos de su función, con mayor precisión que la ecografía endouretral.

resultado del trauma quirúrgico de la prostatectomía radical, o también quizá mediado por lesión neurológica o por pérdida de las propiedades viscoelásticas y de distensibilidad de la uretra esfinteriana a este nivel. Son también son de tener en cuenta las maniobras realizadas postcirugía (dilataciones, exploraciones endoscópicas) y sus consecuencias, en orden a corregir las estenosis de la unión uretro-vesical (más frecuentes de lo reflejado en la literatura).

\section{CONTINENCIA URINARIA POST CIRUGÍA RADICAL DE PRÓSTATA}

Dada la trascendencia de este aspecto, desde la última década han surgido múltiples modificaciones de la técnica quirúrgica $(6,14-17)$, con el objetivo de minimizar esta complicación. La preservación de las fibras del cuello vesical así como una mas depurada técnica quirúrgica ha mejorado notablemente los resultados funcionales actualmente. Por otra parte, el mejor conocimiento de la inervación del esfínter externo, y la más cuidadosa preservación de este en la cirugía mediante nuevas técnicas nos hace que siendo difícil acotar el término incontinencia urinaria post cirugía radical de próstata según su evaluación: "que intensidad" "socialmente aceptable" "cuando, como", sino también secundaria a qué técnica.

\section{Tipo de cirugía}

Hay estudios que proporcionan los resultados funcionales respecto a la continencia urinaria y fase de llenado obtenida en pacientes sometidos a prostatectomía radical laparoscópica y abierta. En líneas generales la continencia urinaria socialmente aceptable se presenta en cerca del $80 \%$ de los casos en centros de excelencia. Hay autores que determinan la continencia urinaria estudiando el perfil uretral dinámico y mediante la cistomanometría. Comparan una serie homogénea de enfermos mediante cirugía laparoscópica / abierta. En los pacientes incontinentes al esfuerzo demuestran tasas anormalmente altas de hiperactividad del detrusor, más altas en el grupo de enfermos sometidos a cirugía abierta. Concluyen que en su experiencia la prostatectomía radical abierta tienen un impacto negativo en la fase de llenado vesical y en la función esfinteriana. No hay realmente en su serie diferencias en la función uretral en el postoperatorio entre la técnica abierta y laparoscópica y piensan que la cirugía laparoscópica proporciona menos alteraciones de la acomodación y la fase de llenado que la laparoscopia para iguales tasas de continencia al esfuerzo (15). Otros autores no tienen esa percepción al respecto como veremos mas adelante.

Respecto a la cirugía Robótica asistida frente a laparoscópica, en lo que se refiere a continencia urinaria hay pocas diferencias globales. Las tasas de continencia global al esfuerzo (16) se cifran en el $83 \%$ frente al $81 \%$, a favor de la prostatectomía laparoscópica sin robot. Esto es así en una serie de $247 / 125$ enfermos respectivamente a 1 año de evolución. Sin embargo, si se introduce el factor severidad de la incontinencia a más de una compresa/ día, los resultados resultan favorables a la cirugía radical robótica-asistida. Concluyen los autores que, a pesar de que el interés de los cirujanos por el robot es evidente, las tasas de continencia a pesar de ser una larga serie precisa resultados a más largo plazo que discrimine estas dos técnicas.

Proceder a la prostatectomía radical robóti$\mathrm{ca}$, ha sido un gran desafío actual en lo que se refiere a la disección del cuello vesical por tener que comenzar una nueva curva de aprendizaje. Weimerg (17) evalúa sus resultados de continencia en 619 prostatectomías radicales laparoscópicas con robot. Se realizó una técnica depurada de disección del cuello vesical, con preservación del mismo frente, a la técnica estándar realizando un estudio comparativo. Evalúan los enfermos a los 4, 12 y 24 meses y concluyen que para la misma tasa de continencia que se aproxima al 100\%, la recuperación de la continencia es más rápida en los enfermos de una forma significativa. 


\section{Volumen prostático}

Los enfermos con cáncer de próstata localizado y un volumen prostático grande, pudieran tener un manejo controvertido. Los resultados de la prostatectomía radical en los pacientes con próstata voluminosa son favorables en lo que se refiere al tumor y comparable a los resultados de continencia y función eréctil que se obtiene en pacientes con próstata pequeña.

En lo que se refiere a tasas de continencia no hay diferencias respecto a pacientes con volúmenes prostáticos pequeños. Además, hay que insistir que la cirugía beneficia particularmente a los enfermos con grandes volúmenes prostáticos, que son los que tienen más síntomas previos lógicamente, sin perjuicio en la continencia (18).

\section{Obesidad}

La incidencia creciente de cáncer de próstata y obesidad hace que el urólogo se deba enfrentar cada vez más frecuentemente a pacientes obesos con cáncer de próstata. Han sido muy estudiados los resultados quirúrgicos en lo que se refiere a continencia urinaria entre pacientes obesos y no obesos sometidos a prostatectomia radical abierta.

Los pacientes obesos sufren en mayor medida incontinencia urinaria y estenosis a nivel vésicouretral que los pacientes no obesos (19). Adicionalmente hay que considerar que la población de pacientes obesos es mucho más frecuente la hiperactividad del detrusor que en los no obesos.

\section{Tiempo desde la cirugía}

El tiempo transcurrido desde la cirugía es otro aspecto a considerar, pues la aparición de continencia, es dependiente de este (14) quizá como consecuencia de la estabilización funcional y la creación de fenómenos de reinervación. También influyen como vernos las maniobras de rehabilitación en el retorno de la continencia. Más allá del año se puede pensar globalmente en la estabilización clínica de la situación.

Glickman (20) estudia los cambios en la recuperación de la continencia urinaria espontánea y de la función eréctil entre 2 y 4 años después de la prostatectomía radical. En lo que se refiere a continencia, sobre la población de incontinentes, del $2^{\circ}$ al $4^{\circ}$ año recupera la continencia un porcentaje pequeño, pero no despreciable $(12,2 \%)$.
Edad

Un estudio relevante y modélico (21) que estudia la continencia urinaria en 1291 pacientes, en los que se ha realizado prostatectomía radical, muestra que la incidencia de incontinencia urinaria de esfuerzo es relevante en el $8,4 \%$ de los pacientes. Al estudiar este grupo de enfermos, se demostró que más allá de los aspectos técnicos, la incontinencia urinaria de esfuerzo era más frecuente con significación estadística en los enfermos de mayor edad, llegando al $14 \%$ en la subpoblación mayor a 70 años..

\section{Resección transuretral previa}

En porcentaje sustancial de varones se detecta de forma incidental o inesperadamente un cáncer de próstata después de una resección transuretral o en su seguimiento evolutivo. Además, la edad y los valores de PSA son factores independientes que pueden predecir aquellos que pueden padecer cáncer de la próstata.

Podríamos pensar que la cirugía radical de próstata en pacientes a los que se les ha practicado una RTU prostática (RTUP) podría tener diferentes tasas de continencia, al haberse afectado el cuello vesical. Puede afectarse físicamente o bien su inervación. También por ello se pudiera afectar su reconstrucción. No está claro como influye dicho extremo

Se ha comparado la morbilidad y los resultados funcionales después de la prostatectomía radical laparoscópica, con y sin resección transuretral previa de la próstata (22). En 640 pacientes se realizó prostatectomía radical laparoscópica, de los cuales $46(7,2 \%)$ habían sido sometidos a RTU de próstata. Los datos perioperatorios y postoperatorios fueron comparados entre el grupo 1 (con la RTUP previa) y el grupo 2 (sin RTUP previa). Los resultados funcionales se evaluaron mediante cuestionarios auto administrados a los 12 y 24 meses después de la cirugía. Dos años después de la cirugía, la tasa de continencia fue $86,9 \%$ en el grupo 1 y $95,8 \%$ en el grupo 2 ( $p=0.77)$, y la tasa de potencia fue de $63,8 \%$ y $70,9 \%$, respectivamente, después de la preservación bilateral de haces neurovasculares $(P=.61)$. Sin embargo, la preservación de haces neurovasculares se realizó después de la RTUP anterior en sólo el 56,5\% del grupo 1 vs $78,9 \%$ en el grupo $2(P=.02)$. La mediana de seguimiento de los enfermos fue de 50,8 meses (rango 30-107).

La prostatectomía radical laparoscópica se puede realizar después de la RTUP sin comprometer los resultados oncológicos. Sin embargo, los pacien- 
tes deben ser informados de que el procedimiento se asocia con peores resultados intra y postoperatorias. Aunque la tasa de continencia urinaria no se ve obstaculizada por la RTUP anterior, la preservación de haces neurovasculares es técnicamente más difícil y compromete la función eréctil postoperatoria.

\section{Tiempo de comienzo y control de reeducación del sue- lo pélvico}

La reeducación del suelo pélvico, juega un papel en la recuperación de la continencia. Parece que no vale solo con dar unas sutiles instrucciones de fortalecimiento muscular al enfermo, hay que documentar dichos ejercicios y monitorizarlos para tener control de resultados de dicha intervención.

En un estudio de casos control noruego, se evalúan dos grupos de pacientes con incontinencia post-prostatectomía desde el tercer mes de intervención ( $\mathrm{A}$ y $B$ ). Ambos grupos recibieron instrucciones de corregir las contracciones musculares del suelo pélvico y se les animó a entrenar dicha musculatura. A uno de los grupos se le ofreció un seguimiento adicional de capacitación instrucciones guiadas por un fisioterapeuta. Después de 6 meses hubo una diferencia clínicamente relevante en el estado de continencia entre los grupos: $79 \%$ eran continentes en el grupo $\mathrm{A}$ y $58 \%$ en el grupo B. Doce meses después de la cirugía, la diferencia fue clínicamente y estadísticamente significativa $(p=0,028)$ en favor de un grupo, y el $92 \%$ eran continentes en el grupo A y $72 \%$ en el grupo B. El grupo que fue asistido por un fisioterapeuta recobró la continencia antes que los pacientes que realizaron ejercicios por su cuenta (23).

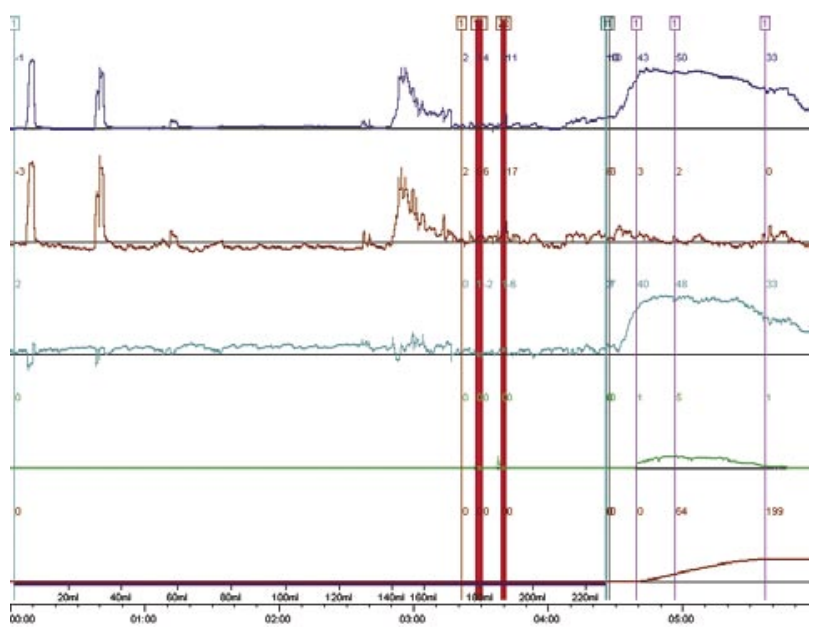

FIGURA 3. Instantánea miccional urodinámica con obstrucción del tracto urinario inferior compresiva.

\section{OBSTRUCCIÓN DEL TRACTO URINARIO INFE- RIOR EN EL CÁNCER DE PRÓSTATA}

La glándula prostática posee unas características que determinan su comportamiento y la sintomatología ocasionada por su crecimiento. En primer lugar la glándula es una estructura anatómica hormonodependiente. Las observaciones de pacientes con alteraciones de la función androgénica sugieren que es precisa la existencia de hormonas sexuales masculinas después de la pubertad al menos para iniciar la hiperplasia estromal y epitelial características de la HBP. Sin embargo, los valores séricos de testosterona no guardan relación con el proceso de crecimiento glandular; más bien se trata de un cambio en el medio homeostático hormonal que acontece cuando el varón envejece, con un aumento de la concentración relativa de estrógenos por mayor conversión periférica en el tejido adiposo, así como valores aumentados de dihidrotestosterona en el tejido prostático.

Aunque HBP y cáncer de próstata presentan etiologías diferentes, ambos procesos aumentan su frecuencia con la edad y posiblemente estén sujetos a influencias comunes, por lo que pueden coexistir en un mismo paciente.

La localización del carcinoma de próstata influye en la posibilidad de desarrollar obstrucción del tracto urinario inferior. No debemos olvidar la posibilidad de frecuente coexistencia con hiperplasia prostática benigna, que puede originar un complejo sintomático característico. Podemos asumir que los tumores localizados en la zona de transición o en su proximidad, ocasionan el mismo proceso compresivo sobre la uretra prostática que el crecimiento benigno

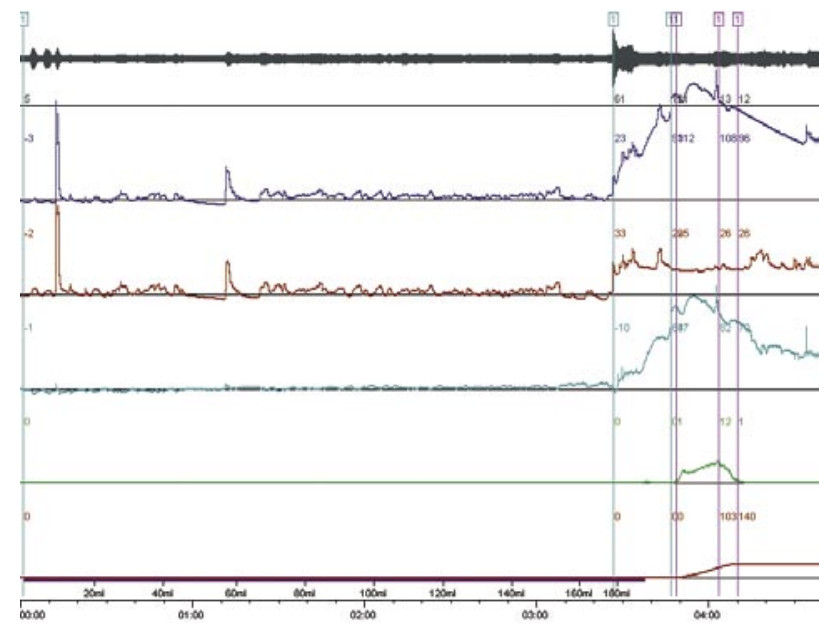

FIGURA 4. Instantánea miccional urodinámica con obstrucción del tracto urinario inferior constrictiva. 
de la próstata, así como un cierto grado de obstrucción constrictiva por obstrucción uretral directa (Figuras 3 y 4$)$.

En otros casos la obstrucción del tracto urinario inferior hace debilitar la función del detrusor precisándose el empleo de prensa abdominal para conseguir el vaciado vesical (Figura 5).

Los síntomas pueden ser evaluados por cuestionarios oficiales diseñados al uso como la escala de Boyarsky o el tipo IPSS. Los síntomas obstructivos obedecen a la compresión-constricción uretral antes mencionada, que provoca aumento en el tiempo de la micción, dificultad de inicio, disminución del calibre y de la fuerza de proyección del chorro miccional. La hipertrofia del músculo detrusor

\section{Esclerosis de la unión cuello vesical - uretra}

Una complicación relativamente frecuente de la prostatectomía radical (mayor de lo referido en la literatura) es la estenosis de la anastomosis uretravesical.

La estenosis de la unión uretra-vesical tiene una horquilla menor que las tasas de continencia en la literatura, variando del 0,4 al $32 \%(24)$.

Los factores que se relacionan a su origen son técnicos o inherentes a complicaciones quirúrgicas. Influye la extravasación, resección transuretral previa, duración del sondaje, ...otros factores como a radioterapia o la diatermia en cuello vesical han sido considerados como factores predisponentes.

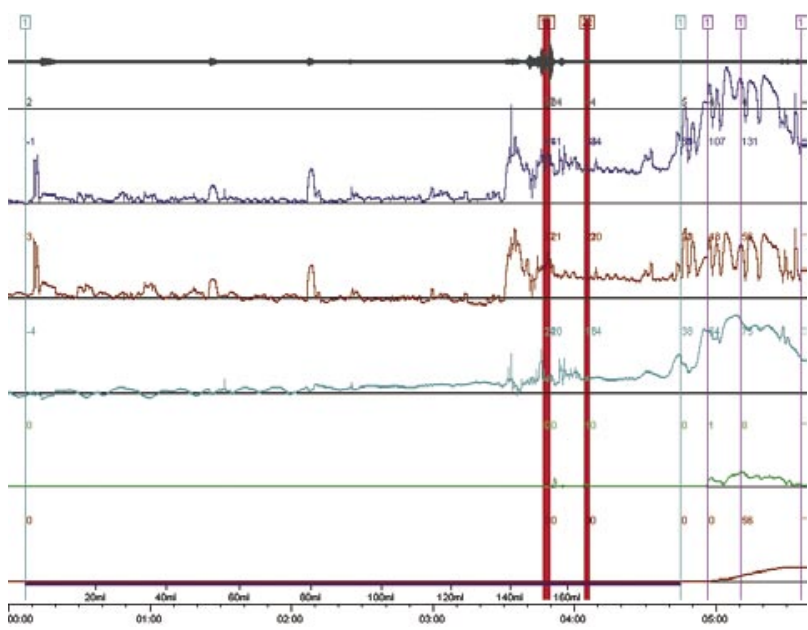

FIGURA 5. Obstrucción del tracto urinario inferior con empleo de prensa abdominal supletoria para conseguir la evacuación urinaria sin residuo post-miccional.
La esclerosis de la anastomosis cuello vesical uretra después de prostatectomía radical abierta se ha informado que ocurre en un 5 a un $32 \%$ de los casos y del 0 al $3 \%$ después de prostatectomía radical laparoscópica. Igualmente volvemos a ver rangos excesivamente amplios y también están.

El cierre óptimo de la anastomosis involucra diversos elementos que contribuyen:

- Aproximación hermética

- Aposición con tensión libre,

- Buena vascularización

- Correcto enfrentamiento mucoso y

- Reordenación adecuada de la uretra

Las causas de la esclerosis de la anastomosis cuello vesical- uretra después de prostatectomía radical son generalmente pobremente entendidas, sin embargo se relacionan a múltiples factores:

- luz excesiva que se re-estrecha en el sitio de la reconstrucción,

- Isquemia del tejido local

- Fallo de aposición mucosa

- Extravasación urinaria

Estos autores estudian la incidencia en la porstatectomía radical laparoscópica robot asistida, en relación al índice de masa corporal, a la edad, a la pérdida sanguínea, asl tiempo quirúrgico al peso de la próstata y al función vesical.

La esclerosis de la anastomosis cuello vesicaluretra se presentó en el 1,1 \% de los casos con un tiempo de aparición de 4 meses promedio y siempre dentro del primer año. Su reparación no tuvo ninguna incidencia en la continencia posterior ni el la calidad de vida. Piensan que esta baja incidencia es dependiente de tener adecuados instrumentos, con una maniobrabilidad mejorada, y la disminución de la pérdida sanguínea que proporciona una mejor visualización (25).

\section{HIPERACTIVIDAD DEL DETRUSOR Y CÁNCER DE PRÓSTATA}

La hiperactividad del detrusor puede presentarse en pacientes con cáncer de próstata en diversas situaciones clínicas y con diferentes condicionantes. Puede aparecer inicialmente de forma concomitante a los síntomas obstructivos en el comienzo de la sintomatología, siendo indistinguible de la que se presenta con la hiperplasia benigna de próstata a veces concomitante, en el seno de la obstrucción del tracto urinario inferior. 


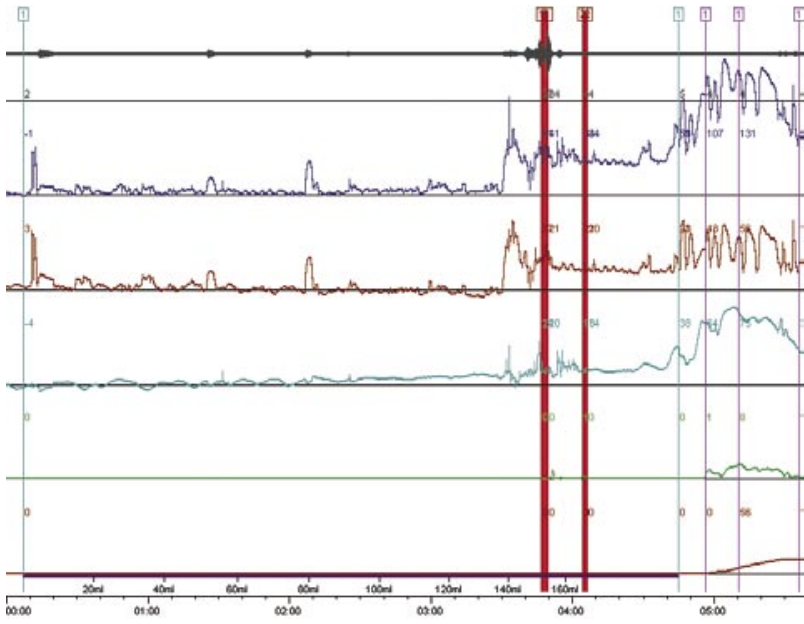

FIGURA 6. Obstrucción del tracto urinario inferior con empleo de prensa abdominal supletoria para conseguir la evacuación urinaria sin residuo post-miccional.

Con la progresión del tumor prostático se pueden presentar variantes pudiéndose observar en algunos casos a condicionar cuadros clínicos de hiperactividad vesical por estímulo local por el crecimiento en volumen del tumor prostático. Con crecimientos avanzados puede presentarse hiperactividad del detrusor de medio ciclo miciconal con importante repercusión funcional por los sintomatología que suele acompañar de urgencia miciconal, micción involuntaria y pérdida del control miccional (Figura 6). El tratamiento quirúrgico desobstructivo puede en ocasiones hacer cambiar el patrón urodinámico, mejorando los síntomas obstructivos, pero manteniéndose

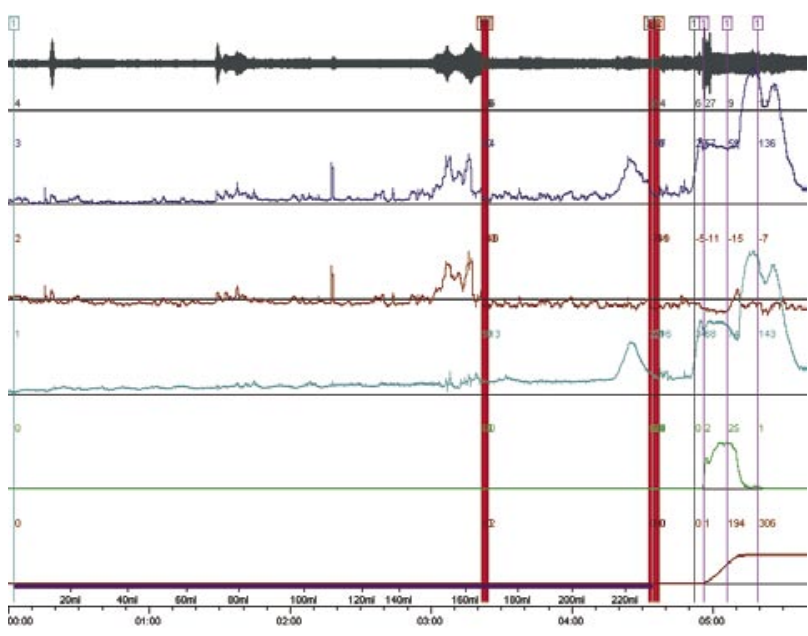

FIGURA 7. El tratamiento quirúrgico demuestra la mejoría de la dificultad miccional pero la persistencia de hiperactividad del detrusor. los irritativos por persistencia de la hiperactividad del detrusor (Figura 7).

Otro patrón urodinámico quer aparece es la acomodación vesical disminuida con el crecimiento del tumor. En algunos casos incluso llegando a la desfuncionalización vesical en estadios avanzados. Comparten estos casos clínicos la obstrucción del tracto urinario inferior, la desfuncionalización del mecanismo esfinteriano, trastornos de la fase de llenado y de la de vaciado (Figura 8).

El patrón urodinámico de baja acomodación vesical es frecuente después de la prostatectomía radical en los primeros tres meses después de la cirugía y retorna rápidamente a la normalidad casi siempre (26).

Se puede ver asociada la hiperactividad vesical después de todas las opciones de tratamiento del tumor órgano-confinado: prostatectomía radical en cualquiera de sus modalidades, abierta, laparoscópica robótica o perineal, post-radioterapia y postbraquiterapia.

Ya hemos visto que algunos autores refieren una mayor frecuencia de hiperactividad vesical después de prostatectomía radical abierta frente a laparoscópica, sin embargo otros muchos autores no refieren tal dato. Sin embargó si que está descrita la hiperactividad del detrusor "de novo" en pacientes en los que se implantó un In Vance en un $5 \%$ de los pacientes (27). También está descrita la hiperactividad "de novo" en 2 de 5 pacientes con problema severo de incontinencia, post sling masculino (28).

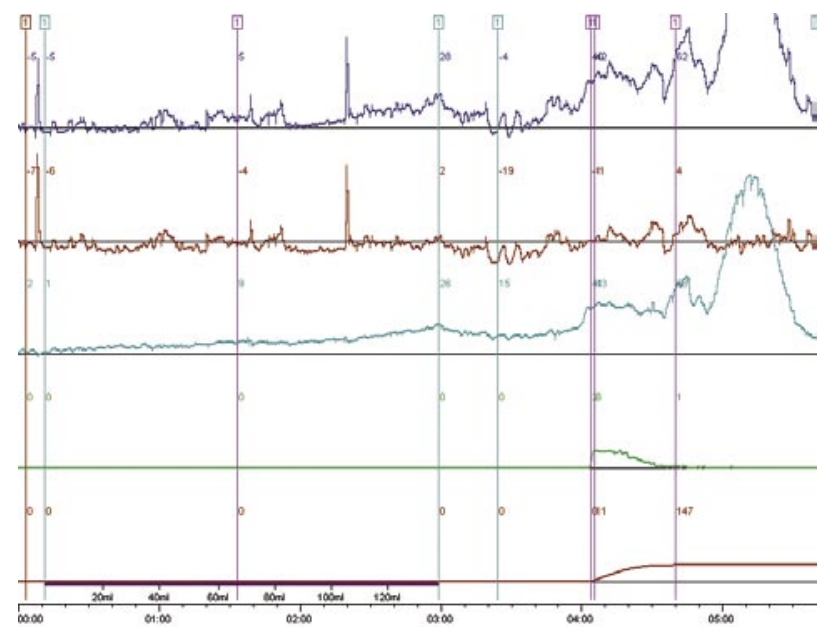

FIGURA 8. Baja acomodación vesical en paciente con cáncer de próstata. 
Existe controversia, si los parámetros urodinámicos condicionan en algún modo el tratamiento de la incontinencia urinaria de esfuerzo secundaria a prostatectomía radical, mediante esfínter urinario artificial o si pueden predecir sus resultados. Creemos que el estudio urodinámico previo es preceptivo al implante de un esfínter, aunque hay autores que lo cuestionan. Está descrito que un $24 \%$ de los enfermos con incontinencia urinaria de esfuerzo secundaria a prostatectomía radical presentan urgencia miciconal. (29). Los datos que nos puede proporcionar el estudio, además de la incontinencia de esfuerzo que podrían suponer factor de fracaso son:

- Demostración de hiperactividad del detrusor

- Obstrucción concomitante

- Primera sensación miccional a baja capacidad

- Baja acomodación vesical,

- Capacidad vesical disminuída

Para estos autores, ninguno de estos factores influye en peores resultados de tratamiento. Indudablemente nosotros pensamos que estos factores sí que tienen trascendencia para el tratamiento de esta patología funcional, más que nada, porque si que cabe un empeoramiento posible en estos casos, después de someterse a una intervención quirúrgica que además es costosa, ha de conocerlo el enfermo previamente.

La radioterapia externa, con un efecto dosisdependiente, suele producir síntomas urinarios obstructivos e irritativos. Se ha estudiado el estado actual de la fisiopatología de los síntomas del tracto urinario inferior (LUTS) secundarios a la radioterapia para el cáncer de próstata y las opciones de tratamiento de esta complicación, sin embargo adolecen los estudios encontrados de evaluación urodinámica de dichos síntomas (30). Se han revisado los efectos inducidos por la radiación en el tracto urinario inferior, que se traducen en LUTS, producidos por la lesión de las neuronas periféricas, fibrosis intersticial del detrusor, y por la pérdida de fibras musculares de la muscular propia. Los LUTS en estos casos se asocian con un deterioro muy importante de la calidad de vida y de la función sexual. Numerosos estudios no aleatorios y un estudio aleatorizado apoyan a la hipótesis de que la aparición de los LUTS secundarios a la radioterapia es efectivamente mitigado por bloqueo de los receptores adrenérgicos alfa 1 . Los estudios básicos y clínicos, así como las directrices clínicas pertinentes para los LUTS secundarios a radioterapia sugieren, que el uso rutinario de alfa l-adrenérgicos deben considerarse en los pacientes tratados con radioterapia, bien como profilaxis o cuando se presenten signos más precoces de dichos síntomas. No está documentada en esta serie la existencia de hiperactividad del detrusor, baja acomodación obstrucción y otras entidades.

Después de la braquiterapia, en un grupo sustancial de pacientes se producen síntomas irritativos en relación a hiperactividad vesical (31). El empleo de anticolinérgicos puede hacer llegar a conseguir la normalización del IPSS en el $79.7 \%$ los pacientes. Doce meses después de la iniciación del tratamiento anticolinérgico, el IPSS había disminuido por una media de 4.3 puntos siendo la mejoría mayoritariamente en la urgencia. No se presentaron diferencias significativas para el residuo postmiccional. Aproximadamente el $80 \%$ de los pacientes con hiperactividad del detrusor por braquiterapia responde favorablemente al los anticolinérgicos con sustancial mejora en el IPSS. Un $20 \%$ de los enfermos no responde, o resulta ineficaz o no los tolera.

\section{TOXICIDAD URINARIA Y TOXICIDAD RADIO- INDUCIDA}

Las múltiples opciones de tratamiento para el adenocarcinoma de próstata están ofreciendo tasas de supervivencia similares, por ello la morbilidad relacionada ha de ser una consideración mayor a la hora de elegir el tratamiento. Los síntomas urinarios después de radioterapia y braquiterapia tienen su propio modelo temporal para su desarrollo y su solución. Este modelo es explicado por la fisiopatología subyacente de la lesión de la radiación en ese enclave.

Los conceptos de toxicidad urinaria y toxicidad radioinducida son fundamentalmente empleados por los especialistas en oncología radioterápica y no han llegado a tener su traslación en conceptos fisiopatológicos más precisos en el ámbito de la urodinámica. La toxicidad radioinducida (32) es un cambio molecular de los tejidos inducido por citokinas que al liberarse como consecuencia de la radiación asocian fenómenos de inflamación aguda, proliferación de fibroblastos, fibrosis secundaria y daño vascular. A nivel tisular esta interacción origina cambios inflamatorios mesenquimales y un remodelamiento anómalo de los tejidos. Esto desencadena los trastornos clínicos de mucositis, fibrosis y disfunción del órgano.

Hay una serie de factores relacionados al paciente que influyen en el desarrollo de toxicidad radioinducida, como son las cirugías abdomino-pélvicas previas, enfermedad vascular periférica, tratamientos anticoagulantes $O$ antiagregantes, hipertensión, diabetes, enfermedades del tejido conectivo, enfermedad inflamatoria intestinal y finalmente la edad. 
Otros factores de riesgo son derivados del tratamiento, de los histogramas Dosis-Volumen, la técnica de tratamiento, su fraccionamiento, movimiento o deformación de los órganos, empleo de fármacos radiosensibilizantes.

La dosis rectal media es uno de los parámetros más empleados para valorar la toxicidad, sin embargo no debemos olvidar que este dato depende estrechamente de la técnica global de tratamiento. Otro parámetro es el volumen de irradiación, la inclusión de ganglios pélvicos en el tratamiento se asocia con una mayor toxicidad intestinal, lógicamente, que si solo es irradiada la próstata y por ello derivan mayores trastornos funcionales.

El concepto de toxicidad urinaria implica una lesión sintomática radioinducida sobre la pared vesical y/o uretra como consecuencia del tratamiento radioterápico, originando los cuadros clínicos:

- Cistitis aguda/tardía

- Hematuria

- Disfunción esfinteriana

- Uretritis

Si bien estos conceptos clínicos son los que son útiles para clasificar la sintomatología de los enfermos, desde el punto de vista funcional se quedan cortos y engloban muchas más situaciones, desde cuadros de baja acomodación vesical hasta bloqueo funcional del sistema esfinteriano, tanto de forma aguda como crónica de aparición posterior en el evolutivo.

En lo que se refiere a la incontinencia urinaria secundaria al tratamiento con radioterapia por cáncer de próstata, se presta cada vez más atención. Suele ser de aparición más tardía que la incontinencia post-quirúrgica, con los mismos problemas de definición del síntoma incontinencia y su clasificación. La incontinencia posterior a radioterapia se ha descrito entre el 0 y el $10 \%$ de pacientes irradiados, sin embargo la percepción subjetiva de los urodinamistas puede ser mayor. Con la introducción de la clasificación más precisa de toxicidad urinaria (32) LENT/SOMA se estima una frecuencia del $1,3 \%$ a 5 años. Si se ajusta a la edad, con el incremento de esta los resultados son menos optimistas. Quizá los especialistas en urodinámica tengan una percepción distinta de estos datos.

En lo que se refiere a la fisiopatología subyacente en los síntomas urinarios, después de braquiterapia por cáncer de próstata, Blaivas (33) estudia una serie de enfermos tratados. Se evalúan 47 enfermos 6 meses después del tratamiento. En 37 ca- sos presentaron hiperactividad vesical relevante, 31 incontinencia urinaria de esfuerzo o de urgencia y 21 presentaron síntomas obstructivos, y disuria persistente en 12 .

Se comparan dos poblaciones una con síntomas persistentes (LUTS) después de braquiterapia, con una población con LUTS sin cáncer de próstata ni braquitrerapia. Los síntomas de los enfermos con braquiterapia difieren de los de la población general, presentando una incidencia mucho más alta de hiperactividad del detrusor, estenosis de uretra y litiasis en uretra prostática.

Algunos candidatos a Braquiterapia por cáncer de próstata localizado presentan una historia previa de resección transuretral prostática, no obstante la RTU origina una cavidad que dificulta técnicamente la braquiterapia. Históricamente esto era una contraindicación para la braquiterapia. Los autores (34) presentan una corta serie de 16 casos tratados sin complicaciones a los 90 días del tratamiento sin alteraciones relevantes en lo que se refiere a continencia. Indudablemente los resultados clínicos funcionales y de supervivencia han mejorado sustancialmente con este tipo de tratamiento.Otros autores (35) presentan su serie con algunas complicaciones pero no establecen que sea una contraindicación.

\section{CALIDAD DE VIDA}

Es fundamental evaluar la calidad de vida en los pacientes con cáncer de próstata porque diferentes tratamientos consiguen resultados de supervivencia similares y frecuentemente es larga.

El urólogo debe reconocer el deterioro en calidad de vida y prestar especialmente atención a ello (4). Ya se ha comentado, y su reiteración obedece a su trascendencia. Este aspecto ha sido investigado a corto y largo plazo la repercusión de los síntomas urinarios intestinales y sexuales en pacientes sometidos a prostatectomía radical radioterapia y braquiterapia. Se emplearon cuestionarios validados SF-36 y el UCLA-PCl en una población de 1366 pacientes desde 1995 a 2007 . En los pacientes tratados por cáncer de próstata localizado se demostró importante discordancia entre la apreciación de calidad de vida por el médico respecto la que manifestó el paciente, sobre todo en lo que se refiere a síntomas urinarios, sexuales intestinales o dolor. La información de los médicos no mejoró en el tiempo.

También se ha evaluado el impacto de la prostatectomía radical (RP) en la calidad de vida (HR$\mathrm{QOL}$ ) en pacientes con edad avanzada con cáncer 
de la próstata (36) Estudian 205 varones de más de 70 años en una evaluación longitudinal. Los pacientes como consecuencia de la prostatectomía radical mostraron problemas físicos que disminuyeron con el tiempo. A los dos años, el $57 \%$ habían regresado a ser asintomáticos. El tiempo de la recuperación de los síntomas urinarios fue de 8.3 meses. Cuando la continencia se definió como "ningún absorbente", el $82 \%$ de pacientes informaron la continencia. Considerando que sólo el $25 \%$ de pacientes recuperaron una función sexual básica, los pacientes mayores seleccionados pueden lograr los resultados funcionales satisfactorios después de la prostatectomía radical.

También se ha estudiado la calidad de vida relacionada a la salud-edad-específica (HRQOL) de pacientes de cáncer de próstata (PCPs) (37). Se analizaron 387 PCPs con el EORTC y con la encuesta QLQ-C30. Los datos obtenidos de los pacientes se compararon con aquéllos de la población alemana general. Los datos HRQOL global recogidos del grupo del estudio frente a la población alemana general son casi iguales sin diferencias. Sin embargo, la mayoría del subdominios de $\mathrm{HRQOL}$ se ven negativamente afectados en los PCPs, y sobre todo en los pacientes más jóvenes (<59 años). Los profesionales médicos deben prestar más atención a la carga emocional de PCPs, porque su tensión y tensiones durante el tiempo antes del tratamiento tienen un bastante más emocional que el carácter físico en muchos casos y no se les presta la debida atención.

Para evaluar la afectación de la calidad de vida en pacientes sometidos a radioterapia a largo plazo se comparó la calidad de vida respecto a síntomas específicos en pacientes 10 años después de ser sometidos a radioterapia externa frente a pacientes vigilados en espera de tratamiento (38). Disminución de calibre miccional, fatiga, nicturia, presentaron algunas diferencias en pacientes sometidos a radioterapia, pero a los 10 años los resultados eran bastante similares demostrándose que la radioterapia tenía una influencia mínima. No se apreciaron diferencias concluyentes en la función eréctil.

\section{BIBLIOGRAFÍA y LECTURAS RECOMENDADAS (*lectura de interés $y^{* *}$ lectura fundamental)}

1. Ferlay J, Autier M, Boniol M, et al. Estimates of the cancer incidence and mortality in Europe in 2006. Ann. Oncol, 2007; 18:851-92.

2. Batista-Miranda JE, Molinuevo B, Pardo Y. Impact of lower urinary tract symptoms on quality of life using functional assessment cancer therapy scale. Urology. 2007; 69(2): 285-8.
3. Tikkinen KA, Auvinen A, Johnson TM $2^{\text {nd }}$, Weiss JP, Keränen T, Tiitinen A, et al. A systematic evaluation of factors associated with nocturia--the population-based FINNO study. Am J Epidemiol. 2009; 170(3):361-8.

**4. Sonn GA, Sadetsky N, Presti JC, Litwin MS. Differing perceptions of quality of life in patients with prostate cancer and their doctors. J Urol. 2009; 15.

5. Steinberg PL, Wason S, Stern JM, Deters L, Kowal B, Seigne J.: YouTube as Source of Prostate Cancer Information.Urology. 2009; 6.

*6. Steiner MS, Morton RA and Walsh PC. Impact of anatomical radical prostatectomy on urinary incontinence. J. Urol, 1991; 145:512-15.

7. Hauri D. Urinary continence after radical prostatectomy: The urodynamic proof of anatomical hypotesis. Urol. Int, 1977; 32:149.

8. Presti JC, Schmidt RA, Narayan PA, Carrol PR and Tanagho EA. Pathophysiology of urinary incontinence after radical prostatectomy. J. Urol, 1990; 143:975-978.

9. Gibbons RP, Correa RJ(Jr), Brannen GE and Mason JT. Total prostatectomy for localized prostatic cancer. J. Urol, 1984; 131:73.

10. Lindner A, de Kernion JB, Smith RB and Kastke FA. Risk of urinary incontinence following radical prostatectomy. J. Urol, 1983; 129:1007.

*11. Mekras JA, Galloway NT, Webster GD, Ramon J, Nandedkar S. The development of a technique to estimate the number of motor units in the urethral sphincter. J. Urol, 1992; 147, 5:1411-5.

12. Prieto L, Jiménez J, Bethencourt F, Dehaini A, Hontoria J, Guil M, et al. Prostatectomía radical: utilidad de la electromiografía del esfínter periuretral en la valoración de la continencia urinaria Arch. Esp. Urol. 47, 5:483-87.

13. Hammerer Rudy DC, Wooside JR and Crawford ED. Urodynamic evaluation of incontinence in patients undergoing modified Campbell radical retropubic prostatectomy: a prospective study. J. Urol, 1984; 132:708.

14. Klein EA. Early continence after radical prostatectomy. J. Urol, 1992; 148, 1:92-5.

**15. Matsukawa Y, Hattori R, Yoshikawa Y, Ono Y, Gotoh M. Laparoscopic versus open radical prostatectomy: urodynamic evaluation of vesicourethral function. Int J Urol. 2009; 16(4):393-6.

*16. Gosseine PN, Mangin P, Leclers F, Cormier L. Pure laparoscopic versus robotic-assisted laparoscopic radical prostatectomy: Comparative study to assess functional urinary outcomes. Prog Urol. 2009;19(9):611-7.

17. Weinberg AC, Lei Y, Soukup JR, Lipsitz SR, Prasad SM, Korkes F, et al. Anatomic bladder neck preservation during robotic-assisted laparoscopic radical prostatectomy: Description of 
technique and outcomes. Freire MP, Eur Urol. 2009 Sep 12.

18. Tal R, Konichezky M, Baniel J. Impact of prostate weight on radical prostatectomy outcomes Isr Med Assoc J. 2009; 11(6):354-8.

19. Van Roermund JG, Van Basten JP, Kiemeney LA, Karthaus HF, Witjes JA. Impact of obesity on surgical outcomes following open radical prostatectomy. Urol Int. 2009; 82(3):256-61.

20. Glickman L, Godoy G, Lepor H. Changes in continence and erectile function between 2 and 4 years after radical prostatectomy. J Urol. 2009; 181(2):731-5.

21. Thiel DD, Young PR, Broderick GA, Heckman MG, Wehle MJ, Igel TC, Petrou SP. Do clinical or urodynamic parameters predict artificial urinary sphincter outcome in post-radical prostatectomy incontinence? Urology. 2007; 69(2):315-9.

22. Menard J, De la Taille A, Hoznek A, Allory Y, Vordos D, Yiou R, Abbou CC, Salomon L L. Laparoscopic radical prostatectomy after transurethral resection of the prostate: surgical and functional outcomes. Urology. 2008; 72(3):593-7.

23. Overgård M, Angelsen A, Lydersen S, Mørkved S. Does physiotherapist-guided pelvic floor muscle training reduce urinary incontinence after radical prostatectomy? A randomised controlled trial.Eur Urol. 2008; 54(2):438-48.

24. Besarani D, Amoroso P, Kirby R. Bladder neck contracture after radical retropubic prostatectomy. BJU Int, 2004; 94:1245.

25. Msezane LP, Reynolds WS, Gofrit ON, Shalhav AL, Zagaja GP, Zorn KC. Bladder neck contracture after robot-assisted laparoscopic radical prostatectomy: evaluation of incidence and risk factors and impact on urinary function. J Endourol. 2008; 22(2):377-83.

26. Natsume O, Kondo H, Cho M, Fujimoto K, Ozono S, Hirao Y.: The impact of radical prostatectomy on patient well-being: a prospective urodynamic study focused on detrusor function. Hinyokika Kiyo. 2004; 50(1):1-6.

27. Giberti C, Gallo F, Schenone M, Cortese P, Ninotta $\mathrm{G}$. The bone anchor suburethral synthetic sling for iatrogenic male incontinence: critical evaluation at a mean 3-year follow-up. J Urol. 2009; 181(5):2204-8.

28. Ullrich NF, Comiter CV. The male sling for stress urinary incontinence: urodynamic and subjective assessment.J. Urol. 2004; 172(1):204-6.
29. Thiel DD, Young PR, Broderick GA, Heckman MG, Wehle MJ, Igel TC, Petrou SP. Do clinical or urodynamic parameters predict artificial urinary sphincter outcome in post-radical prostatectomy incontinence? Urology. 2007; 69(2):315-9.

30. Crawford ED, Kavanagh BD. The role of alphablockers in the management of lower urinary tract symptoms in prostate cancer patients treated with radiation therapy. Am J Clin Oncol. 2006; 29(5):517-23.

31. Bittner N, Merrick GS, Brammer S, Niehaus A, Wallner KE, Butler WM, Allen ZA, Galbreath RW : Role of trospium chloride in brachytherapy-related detrusor overactivity. Urology. 2008; 71(3):460-4.

32. Zapatero A. Efectos secundarios de la RDT radical en el CP. En Calidad de vida en cáncer de próstata.Ed. Por Frances Casas. Ed.Visto Bueno Equipo Creativo. Madrid, 2008. Cap. 3 , pp 39-57.

**33. Blaivas JG, Weiss JP, Jones M.: The pathophysiology of lower urinary tract symptoms after brachytherapy for prostate cancer. BJU Int. 2006; 98(6):1233-7; discussion 1237

34. Merrick GS, Butler WM, Wallner KE, Galbreath RW.: Effect of transurethral resection on urinary quality of life after permanent prostate brachytherapy. Int J Radiat Oncol Biol Phys. 2004; 58(1):81-8.

35. Claros DR, Correia JA, Ferreira LR, Vaz FP.: Retrospective evaluation of urinary continence in patients submitted to brachytherapy after transurethral resection of the prostate gland. Actas Urol Esp. 2009; 33(4):356-60.

36. Namiki S, Ishidoya $\mathrm{S}$, Tochigi $\mathrm{T}$, Ito A, Arai Y. Quality of life after radical prostatectomy in elderly men. Int J Urol. 2009; 16(10):813-9.

37. Zenger M, Hinz A, Stolzenburg JU, Rabenalt R, Schwalenberg T, Schwarz R. Health-related quality of life of prostate cancer patients compared to the general German population: age-specific results. Urol Int. 2009; 83(2):166-70.

38. Fransson P, Damber JE, Widmark A. Health-related quality of life 10 years after external beam radiotherapy or watchful waiting in patients with localized prostate cancer. Scand J Urol Nephrol. 2009; 43(2):119-26. 